\title{
The roles of platelet-derived growth factors and their receptors in brain radiation necrosis
}

\author{
Tomo Miyata', Taichiro Toho', Naosuke Nonoguchi', Motomasa Furuse1', Hiroko Kuwabara², Erina Yoritsune ${ }^{1}$, \\ Shinji Kawabata', Toshihiko Kuroiwa ${ }^{1}$ and Shin-Ichi Miyatake ${ }^{1 *}$
}

\begin{abstract}
Background: Brain radiation necrosis (RN) occurring after radiotherapy is a serious complication. We and others have performed several treatments for RN, using anticoagulants, corticosteroids, surgical resection and bevacizumab. However, the mechanisms underlying RN have not yet been completely elucidated. For more than a decade, platelet-derived growth factors (PDGFs) and their receptors (PDGFRs) have been extensively studied in many biological processes. These proteins influence a wide range of biological responses and participate in many normal and pathological conditions. In this study, we demonstrated that PDGF isoforms (PDGF-A, B, C, and D) and PDGFRs (PDGFR- $\alpha$ and $\beta$ ) are involved in the pathogenesis of human brain RN. We speculated on their roles, with a focus on their potential involvement in angiogenesis and inflammation in RN.

Methods: Seven surgical specimens of RN, obtained from 2006 to 2013 at our department, were subjected to histopathological analyses and stained with hematoxylin and eosin. We qualitatively analyzed the protein expression of each isoform of PDGF by immunohistochemistry. We also examined their expression with double immunofluorescence.
\end{abstract}

Results: All PDGFs were expressed in macrophages, microglia, and endothelial cells in the boundary of the core of $\mathrm{RN}$, namely, the perinecrotic area (PN), as well as in undamaged brain tissue (UB). PDGF-C, D and PDGFR-a were also expressed in reactive astrocytes in PN. PDGFs and PDGFR-a were scarcely detected in UB, but PDGFR- $\beta$ was specifically expressed in endothelial cells not only in PN but also in UB.

Conclusions: PDGFs/PDGFRs play critical roles in angiogenesis and possibly in inflammation, and they contribute to the pathogenesis of RN, irrespective of the original tumor pathology and applied radiation modality. Treatments for the inhibition of PDGF-C, PDGF-D, and PDGFR-a may provide new approaches for the treatment of RN induced by common radiation therapies.

Keywords: Angiogenesis, Brain radiation necrosis, Inflammation, Platelet-derived growth factors, Platelet-derived growth factor receptors

\section{Background}

Higher radiation doses to tumors result in good local tumor control and improvement in overall survival. On the other hand, radiation necrosis (RN) in the brain occurring after radiotherapy for brain tumors as well as for head and neck cancers is a serious complication that decreases the quality of life in patients. The mechanisms underlying $\mathrm{RN}$ have not been completely elucidated. In a

\footnotetext{
* Correspondence: neu070@poh.osaka-med.ac.jp

'Department of Neurosurgery, Osaka Medical College, 2-7 Daigaku-machi, Takatsuki City, Osaka 569-8686, Japan

Full list of author information is available at the end of the article
}

previous study we showed that RN specimens stained with hematoxylin and eosin (H\&E) typically show marked angiogenesis, so-called telangiectasis, microbleeding, and interstitial edema, probably caused by leakage of plasma from leaky angiogenesis into the surrounding necrotic core-namely, the perinecrotic area (PN) [1].

We and others have applied several treatments for RN, such as anticoagulants, vitamin E, corticosteroids, and surgical resection [2-4]. The typical MRI of symptomatic RN from case 3 demonstrated rapid shrinkage of the perilesional edema after surgical treatment [see Additional file 1 and Table 1]. After surgical resection for the only 
Table 1 Clinical features of patients with symptomatic radiation necrosis

\begin{tabular}{|c|c|c|c|c|c|c|c|}
\hline Pt. & Age (y) & Sex & Original dis. & Radiation $^{a}$ & Resection area (lobe) & Duration $^{b}$ & Chemo \\
\hline 1 & 46 & $\mathrm{~F}$ & SCC. & XRT (60 Gy), BNCT (13.9 Gy-Eq) & Temporal & 7 & MTX \\
\hline 2 & 78 & M & Sal. Duc. Ca. & XRT (60 Gy), BNCT (13.9 Gy-Eq) & Frontotemporal & 20 & - \\
\hline 3 & 18 & M & GBM & XRT (IMRT) (74 Gy) & Parietal & 37 & - \\
\hline 4 & 63 & $\mathrm{~F}$ & GBM & XRT (24 Gy), BNCT (13 Gy-Eq) & Frontoparietal & 4 & - \\
\hline 5 & 34 & M & GBM & XRT (24 Gy), BNCT (13 Gy-Eq) & Frontal & 6 & - \\
\hline 6 & 56 & $\mathrm{~F}$ & GBM & Proton + XRT (total 90 Gy) & Temporoparietal & 10 & ACNU \\
\hline 7 & 46 & $\mathrm{~F}$ & Ade. Ca. & XRT (30 Gy), SRS (55 Gy, 65 Gy) & Frontal & 32 & Herceptin \\
\hline
\end{tabular}

Pt, patient; $y$, year; F, female; $M$, male; Original dis, original disease; SCC, Squamous cell carcinoma; Sal. Duc. Ca, salivary ductal carcinoma; GBM, glioblastoma; Ade. $\mathrm{C} a$, adenocarcinoma; XRT, X-ray radiation treatment; IMRT ,intensity modulated radiation therapy; $B N C T$, boron neutron capture therapy; Proton, proton beam therapy; MTX, methotrexate; ACNU, nimustin; Herceptin, trastuzumab;

In Pt. 1 and 2, the temporal lobe was included in the irradiation field and in Pt. 3, 4, 5, and 6, local radiation therapy was administered. Pt. 7 had received whole brain irradiation and received SRS twice. In BNCT, the presented dose is the peak point dose for the normal brain.

${ }^{\mathrm{b}}$ Months between termination of the last radiotherapy and onset of symptoms caused by radiation necrosis.

enhanced lesion, the perilesional edema decreased rapidly compared with preoperative MRI. This rapid shrinkage of the perilesional edema after surgical treatment was also observed in other cases. In addition, bevacizumab, an antibody for vascular endothelial growth factor (VEGF), has recently shown promising effects on symptomatic brain $\mathrm{RN}$ and symptomatic pseudo-progression [5,6]. However, in some cases, treatment with bevacizumab was not sufficient to resolve RN. Some RN cases recurred as RN even after temporary remission by bevacizumab treatment [7].

Recent experiments have shown that demyelination and damage of the normal vasculatures and the appearance of abnormal vasculatures around necrotic foci are major issues in the development of RN [8,9]. In addition, we previously reported that hypoxia-inducible factor $1 \alpha$ $(\mathrm{HIF}-1 \alpha)$ and VEGF are key molecules in RN [1]. In a later study, we tried to determine whether not only HIF$1 \alpha$ and VEGF, but also proinflammatory cytokines such as IL-1 $\alpha$, IL-6, TNF- $\alpha$, and NFKB, might play significant roles in RN, since these cytokines were produced by CD68and hGLUT5-positive microglia and/or macrophages accumulated in PN (in submission).

The platelet-derived growth factors (PDGFs) signaling pathway, which has been extensively studied and shown to play critical roles in many biological processes, is mediated through tyrosine kinase receptors (PDGFR- $\alpha$, PDGFR- $\beta$ ) $[10,11]$. There are five members of the PDGF family: PDGF-A, B, and $\mathrm{AB}$, and the recently discovered PDGF-C and D. So far, no heterodimers involving the PDGF-C and D chains have been described. PDGF-A binds only PDGFR- $\alpha$, whereas PDGF-B activates PDGFR- $\alpha$, $\alpha \beta$, and $\beta$. PDGF-A, B, and C activate PDGFR- $\alpha$ and $\alpha \beta$, while PDGF-D specifically binds to and activates its cognate receptor PDGFR- $\beta$. In other words, according to published data, PDGFR- $\alpha$ binds PDGF-A, B, AB, and C, whereas PDGFR- $\beta$ binds PDGF-B and D $[10,12,13]$.

In addition, PDGF- $A$ and $B$ are secreted in their active forms, while PDGF-C and D are secreted as inactive forms requiring activation for their function [14]. Interestingly, several reports have shown that the structure and biological function of PDGFs are quite similar to those of VEGF [15]. Therefore, the PDGF family is sometimes referred to as the VEGF family. Nevertheless, in recent years it was revealed that the angiogenic pathway induced by PDGF-C is, in large part, VEGF-independent [16].

Based on these findings, in this retrospective study we performed histopathological and immunohistochemical analyses on 7 human RN specimens from patients who we had treated surgically from 2006 to 2013 at our department. We here describe the findings common to all 7 of these specimens, and demonstrate which type of cells produce PDGFs and which type express the PDGFRs. We also evaluated the roles of PDGFs/PDGFRs in brain RN.

\section{Methods}

\section{Case selection}

Seven surgical specimens, obtained from 2006 to 2013, were submitted for histopathological analysis, staining with $H \& E$, and immunohistochemistry. All the patients had received radiotherapy, including X-ray treatment (XRT), stereotactic radiosurgery (SRS), proton beam therapy, and boron neutron capture therapy (BNCT). The primary diseases were 4 glioblastomas, 2 head and neck cancers, and 1 metastatic brain tumor derived from breast cancer.

In this study we selected the area as radiation necrosis with extensive necrotic area with the boundary of extensive angiogenesis and edema, which is continuous to undamaged brain tissue, as mentioned in Background.

For the 2 patients with head and neck cancers, radiotherapy was used to treat the parotid lesions and the temporal lobe was included in the irradiation field. Therefore, there were no tumor cells in the brain, indicating pure brain RN. The patient characteristics are detailed in Table 1. 


\section{Histological and immunohistochemical staining}

Histological and immunohistochemical analyses were performed on paraffin sections in which we observed the presence of RN by H\&E staining. Each section was immunostained with the following antibodies: PDGF-A (1:20; R\&D Systems, USA), PDGF-B (1:20; Abcam, Japan), PDGF-C (1:100; R\&D Systems), PDGF-D (1:50; R\&D Systems), PDGFR- $\alpha$ (1:20; R\&D Systems), and PDGFR- $\beta$ (1:50; R\&D Systems) (Table 2). We routinely use a pressure cooker for 4 minutes to retrieve all the antigens. Endogenous peroxidase was blocked with $0.03 \%$ hydrogen peroxide for 40 minutes at room temperature. We used the $\mathrm{ABC}$ technique (Vector Laboratories, USA) for all of these antigens, before DAB (3, 3' diaminobenzidine tetrahydrochloride (Wako Pure Chemical Industries, Japan)). The sections were counterstained with hematoxylin 3G (Sakura Finetek, Japan) and mounted.

\section{Immunofluorescence}

Double immunofluorescence was performed using the following antibody combinations: PDGF-C and GFAP (1:25; Dako, Denmark), CD68 (1:25; Epitomics, USA), hGLUT5 (1:50; IBL, Japan), or CD45 (1:50; Epitomics); PDGF-D and GFAP, CD68, hFLUT5, or CD45; PDGFR- $\alpha$ and GFAP, CD68, hGLUT5, or CD31 (1:20; Dako, Denmark); and PDGFR- $\beta$ and GFAP, CD68, hGLUT5, or CD31.

GFAP, CD68, hGLUT5, CD45, and CD31 were adopted as markers for astrocytes, monocytes, microglia, lymphocytes, and endothelial cells, respectively. All sections were incubated with their respective antibodies for 24 hours with CD68, hGLUT5, and GFAP, and for 48 hours with PDGF-A, B, C, D, and PDGFR- $\alpha$ and $\beta$. Then, after washing the primary antibodies, Alexa Fluor 488 (1:25;

Table 2 List of primary antibodies used

\begin{tabular}{lllll}
\hline Antibody & Clone & Sources & Type & Dilution \\
\hline PDGF-A & & R\&D Systems, & $\mathrm{p} / \mathrm{g}$ & $1: 20$ \\
& & Minneapolis, MN & & \\
PDGF-B & MM0014-5 F66 & Abcam Cambridge, MA & $\mathrm{m} / \mathrm{m}$ & $1: 20$ \\
PDGF-C & R\&D Systems, & $\mathrm{p} / \mathrm{g}$ & $1: 100$ \\
& Minneapolis, MN & & \\
PDGF-D & R\&D Systems, & $\mathrm{p} / \mathrm{g}$ & $1: 50$ \\
& & Minneapolis, MN & & \\
PDGFR-a & & R\&D Systems, & $\mathrm{p} / \mathrm{g}$ & $1: 20$ \\
& & Minneapolis, MN & & \\
PDGFR- $\beta$ & & R\&D Systems, & $\mathrm{p} / \mathrm{g}$ & $1: 50$ \\
& & Minneapolis, MN & & \\
CD68 & KP-1 & Dako, Glostrup, Denmark & $\mathrm{m} / \mathrm{m}$ & $1: 25$ \\
hGLUT5 & & IBL, Tokyo, Japan & $\mathrm{p} / \mathrm{r}$ & $1: 50$ \\
GFAP & 6 F2 & Dako, Glostrup, Denmark & $\mathrm{m} / \mathrm{m}$ & $1: 25$ \\
CD45 & EP322Y & Eptomics, Burlingame, CA & $\mathrm{m} / \mathrm{r}$ & $1: 50$ \\
CD31 & JC70A & Dako, Glostrup, Denmark & $\mathrm{m} / \mathrm{m}$ & $1: 20$ \\
\hline P/g poyclon & & &
\end{tabular}

$\mathrm{p} / \mathrm{g}$ polyclonal goat; $p / r$, polyclonal rabbit; $\mathrm{m} / \mathrm{r}$, monoclonal rabbit; $\mathrm{m} / \mathrm{m}$, monoclonal mouse.
Molecular Probes, USA) or Alexa Fluor 546 (1:25; Molecular Probes) was used (Table 3). Finally, the sections were examined using an LSM510 laser scanning confocal microscope (Carl Zeiss, Germany).

\section{Statistical analysis}

We assessed the frequency of expression of PDGFs semi-quantitatively by the following method. Five fields of each PDGF isoform in which abnormal angiogenesis was detected were randomly selected with a microscope. PDGF-positive mononuclear cells were counted. We observed 7 cases and, to reduce bias, used two observers to count the cells. One observer, who was blinded to the patients' clinical and pathological information, evaluated the results of immunohistochemical staining. The ratios of PDGF-positive cells per total cells in each field were calculated, and we statistically analyzed the data with Steel-Dwass tests using JMP Pro 10 (SAS Institute, USA). The results revealed that PDGF-C and D showed higher frequency of expression than PDGF-A and B in $\mathrm{PN}$. The difference was statistically significant.

\section{Ethical approval}

This study was approved by an institutional committee of Osaka Medical College. The research was in compliance with the Helsinki Declaration.

\section{Results}

\section{Expression of PDGFs}

Figure 1 shows the results of H\&E staining and immunohistochemistry from case 1 . H\&E staining revealed a necrotic

Table 3 Double immunofluorescence combinations

\begin{tabular}{|c|c|c|c|c|c|}
\hline Primary & Dilution & Secondary & Primary & Dilution & Secondary \\
\hline PDGF-C & $1: 50$ & F488 & CD68 & $1: 25$ & F546 \\
\hline PDGF-C & $1: 50$ & F488 & hGLUT5 & $1: 50$ & F546 \\
\hline PDGF-C & $1: 50$ & F488 & GFAP & $1: 25$ & F546 \\
\hline PDGF-C & $1: 50$ & F488 & CD45 & $1: 50$ & F546 \\
\hline PDGF-D & $1: 20$ & F488 & CD68 & $1: 25$ & F546 \\
\hline PDGF-D & $1: 20$ & F488 & hGLUT5 & $1: 50$ & F546 \\
\hline PDGF-D & $1: 20$ & F488 & GFAP & $1: 25$ & F546 \\
\hline PDGF-D & $1: 20$ & F488 & CD45 & $1: 50$ & F546 \\
\hline PDGFR-a & $1: 10$ & F488 & CD68 & $1: 25$ & F546 \\
\hline PDGFR-a & $1: 10$ & F488 & hGLUT5 & $1: 50$ & F546 \\
\hline PDGFR-a & $1: 10$ & F488 & GFAP & $1: 25$ & F546 \\
\hline PDGFR-a & $1: 10$ & F488 & CD31 & $1: 20$ & F546 \\
\hline PDGFR- $\beta$ & $1: 20$ & F488 & CD68 & $1: 25$ & F546 \\
\hline PDGFR- $\beta$ & $1: 20$ & F488 & hGLUT5 & $1: 50$ & F546 \\
\hline PDGFR- $\beta$ & $1: 20$ & F488 & GFAP & $1: 25$ & F546 \\
\hline PDGFR- $\beta$ & $1: 20$ & F488 & CD31 & $1: 20$ & F546 \\
\hline
\end{tabular}

Primary, primary antibody; Secondary, secondary antibody; F488, Alexa Fluor 488; F546, Alexa Fluor 546. 

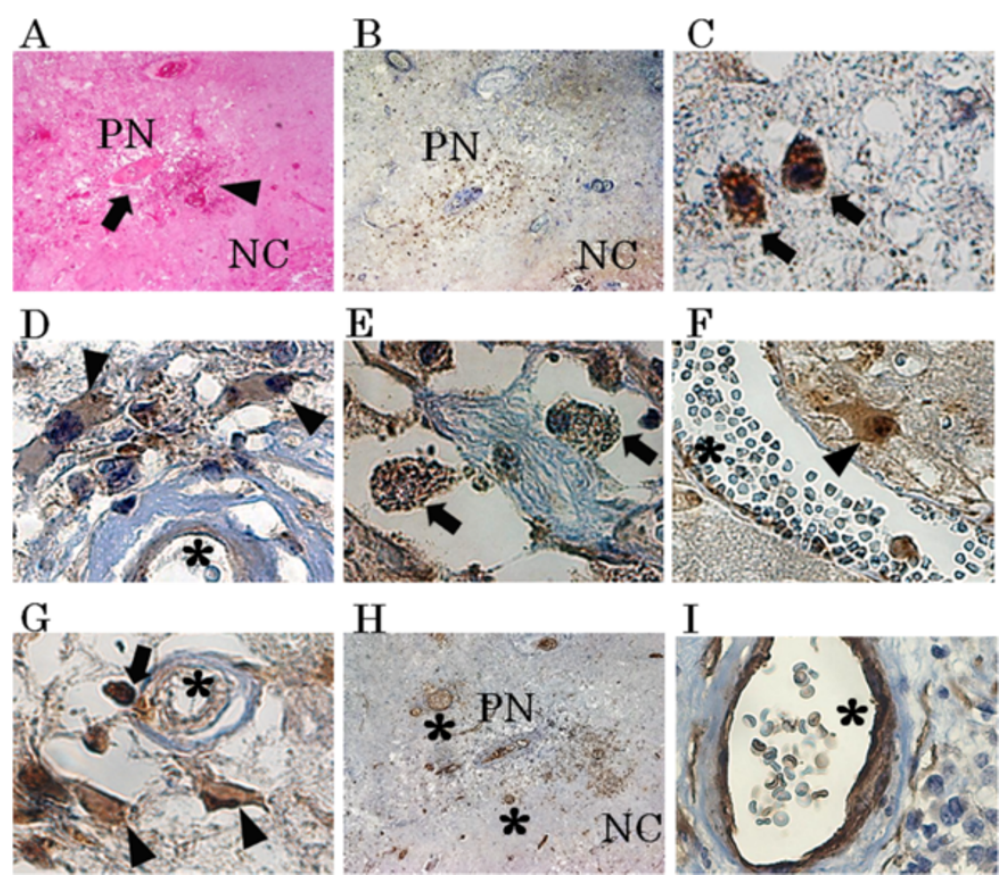

$\mathrm{H}$

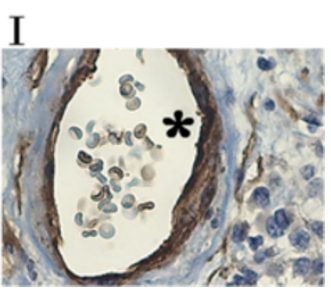

Figure 1 Results of hematoxylin and eosin staining (H\&E) and immunohistochemistry from case 1. H\&E staining (A) revealed a necrotic core (NC) and perinecrotic area (PN), including micro bleeding (A, arrowhead) and abnormal angiogenesis (A, arrow). Immunostaining results for PDGF-C are presented as a representative example (B). PDGF-C (C and D), D (E and F) and PDGFR-a (G) were produced by monocytic cells $(\mathbf{C}, \mathbf{E}, \mathbf{G}$, arrow) and reactive astrocytic cells (D, F, G, arrowhead) in PN. On the other hand, PDGFR- $\beta$ ( $\mathbf{H}$ and $\mathbf{I})$ was expressed mainly in endothelial cells $\left(\mathbf{H}\right.$ and $\mathbf{I}^{*}$ ). There was partially nonspecific staining in $\mathrm{NC}(\mathbf{B})$ or around blood vessels (I). Original magnification, $\mathbf{A}, \mathbf{B}$ and $\mathbf{H} \times 40, \mathbf{C}, \mathbf{D}, \mathbf{E}, \mathbf{F}$, $\mathbf{G}$ and $\mathbf{I} \times 200$.

core (NC) (Figure 1A. NC) and PN (Figure 1A. PN), in which micro bleeding (Figure 1A. arrowhead) and abnormal angiogenesis (Figure 1A. arrow) were confirmed. PDGF-A, B, C, and D-positive cells were detected in PN. The results of immunostaining for PDGF-C are shown as a typical example of these distribution analyses (Figure 1B, C, D). Morphologically, PDGF-A and B were produced by some monocytic cells [see Additional file 2] in PN. On the other hand, PDGF-C and D (Figure 1E, F) were produced by many monocytic cells (arrows in Figure $1 \mathrm{C}, \mathrm{E}$ ), reactive astrocytic cells (arrowheads in Figure 1D, F), and endothelial cells (Figure 1D*). PDGF-A, B, C, and D were scarcely detectable in UB (Figure 2).

These relationships among the expression of PDGFs are summarized in Table 4. These relationships were also confirmed with other specimens [see Additional file 3].

Our statistical analysis revealed that PDGF-C and D showed higher frequencies of expression than PDGF-A and $\mathrm{B}$ in PN. The difference was statistically significant ( $p<0.0001$, Steel-Dwass test) (Figure 3). We also grouped the cases into a GBM group (cases 3, 4, 5, 6) and nonGBM group (cases 1, 2, 7) and analyzed the differences in protein expression between them. No statistically significant differences in the expression of any of the isoforms were observed between the two groups by the Steel-Dwass test [see Additional file 4]. Therefore, we considered that these primary diseases did not affect the expression of PDGFs.

Double immunofluorescence from case 1 revealed that PDGF-C or D-positive cells were merged with many cells positive for CD68 (Figure 4A, E), GFAP (Figure 4B, F), hGLUT5 (Figure 4C, G), and CD45 (Figure 4D, H).

H\&E staining, immunohistochemistry, and double immunofluorescence also showed similar tendencies in other specimens with symptomatic RN [see Additional files 3, and 5].

\section{Expression of PDGFRs}

PDGFR- $\alpha$ was expressed in endothelial cells (Figure 1G*), monocytic cells (Figure $1 \mathrm{G}$ arrow), and reactive astrocytic cells (Figure 1G, arrowhead) in PN. PDGFR- $\beta$ was expressed mainly in endothelial cells (Figure $1 \mathrm{H}, \mathrm{I}^{*}$ ). PDGFR- $\alpha$ was not expressed in any types of cells in UB (Figure 2E), but PDGFR- $\beta$ was detected in endothelial cells in both PN and UB (Figure 2F).

Double immunofluorescence revealed that PDGFR- $\alpha$ and $\beta$ were strongly expressed in CD31-positive cells (Figure 5D, I). PDGFR- $\beta$-positive cells were merged specifically with endothelial cells (Figure 5F, G, H, I and J, *), but PDGFR- $\alpha$-positive cells were merged with cells positive for CD68 (Figure 5A), GFAP (Figure 5B), hGLUT5 (Figure 5C), and CD45 (Figure 5E) in PN. 


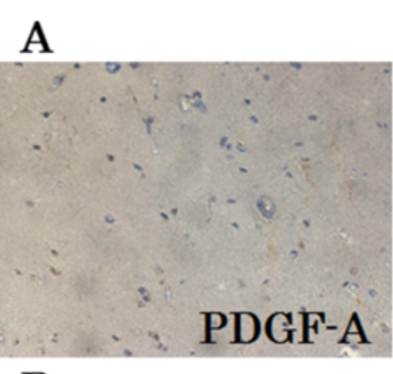

\section{B}

C

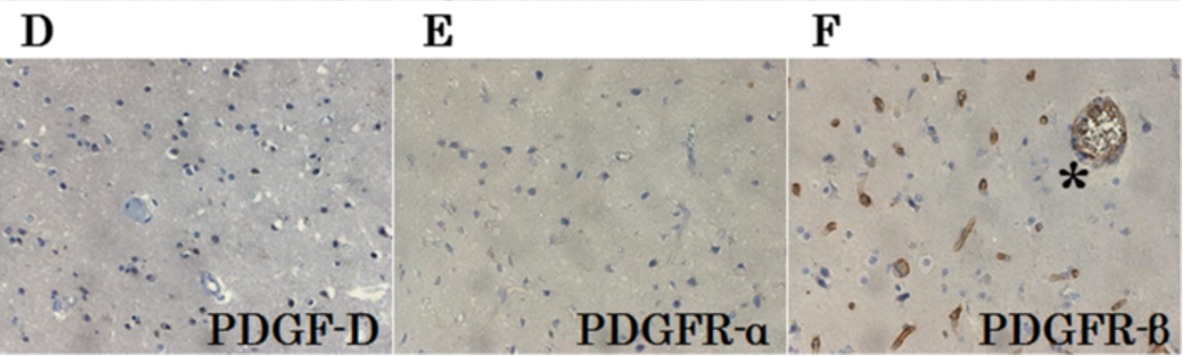

Figure 2 Representative results of immunostaining of undamaged brain tissue (UB). PDGF-A, B, C, D and PDGFR-a were scarcely detectable in UB (A through E). PDGFR- $\beta$ (F) was specifically expressed in endothelial cells in UB. Many normal cerebral blood vessels stained with PDGFR- $\beta$ $\left(\mathbf{F}^{*}\right)$ were detected in UB. Original magnification, $\times 200$.

These findings from case 1 were confirmed in other specimens with symptomatic RN [see Additional file 6].

Double immunofluorescence revealed partially nonspecific staining, especially in endothelial cells (Figures 4 , and $\left.5^{*}\right)$. In cases where immunofluorescence was performed with GFAP alone, vascular endothelial cells were not stained [see Additional file 7]. These findings were also observed in other specimens.

\section{Discussion}

PDGFs are a group of multifunctional proteins with a wide variety of effects. They have important physiologic functions in embryonic and organ development, have been implicated in a wide variety of pathological processes, including proliferation, differentiation, and fibrogenesis, and are essential for the stability of normal blood vessel formation [16-19]. However, the overexpression of

Table 4 Expression of PDGFs/PDGFRs in two areas of the brain

\begin{tabular}{llllllll}
\hline & \multicolumn{1}{l}{ UB } & & & PN \\
\cline { 2 - 4 } \cline { 6 - 7 } & Mono & Astro & Endo & & Mono & Astro & Endo \\
\hline PDGF-A & - & - & - & + & - & + \\
PDGF-B & - & - & - & + & - & + \\
PDGF-C & - & - & - & + & + & + \\
PDGF-D & - & - & - & + & + & + \\
PDGFR-a & - & - & - & + & + & + \\
PDGFR- $\beta$ & - & - & + & & - & + \\
\hline
\end{tabular}

$U B$, undamaged brain area; $P N$, perinecrotic area; Mono, monocytes, including macrophages, microglia and lymphocytes Astro, reactive astrocytes; Endo, endothelial cells ; -, not expressed; +, expressed.

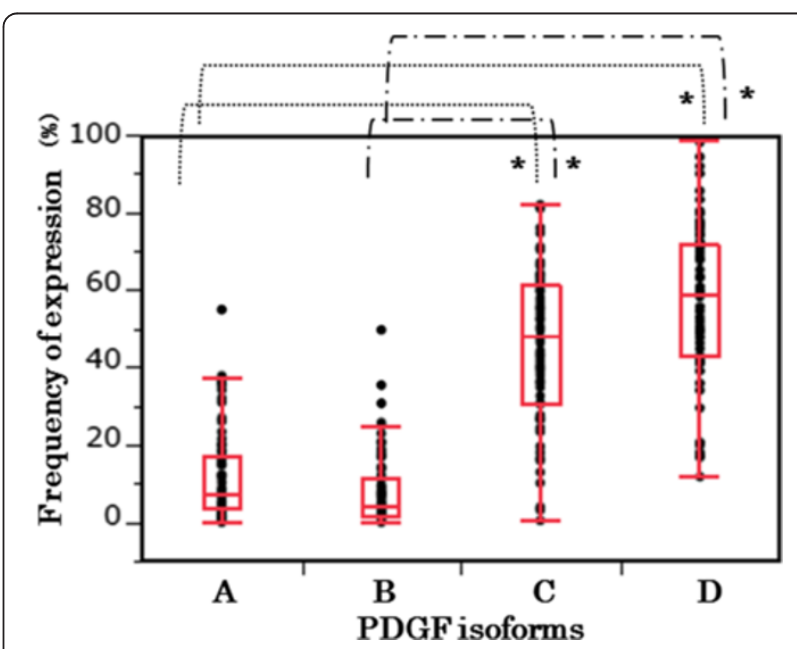

Figure 3 Frequency of expression. We assessed the frequency of expression of PDGFs semi-quantitatively by the following method. Five fields of each PDGF isoform, in which abnormal angiogenesis were detected, were randomly selected with a microscope. The PDGF-positive mononuclear cells were counted. We observed all 7 cases and performed the counting using two observers to reduce bias. One observer, who was blind to the patients' clinical and pathological information, evaluated the results of the immunohistochemical staining. The ratios of PDGF-positive cells to total cells in each field were calculated and were statistically analyzed using Steel-Dwass tests with JMP Pro 10 (SAS Institute Inc., Cary, NC, USA). Statistical analysis revealed that PDGF-C and D showed higher frequency of expression in the PN specimens than did PDGF-A and B. The difference was statistically significant $\left({ }^{*} p<0.0001\right.$,

Steel-Dwass test). 


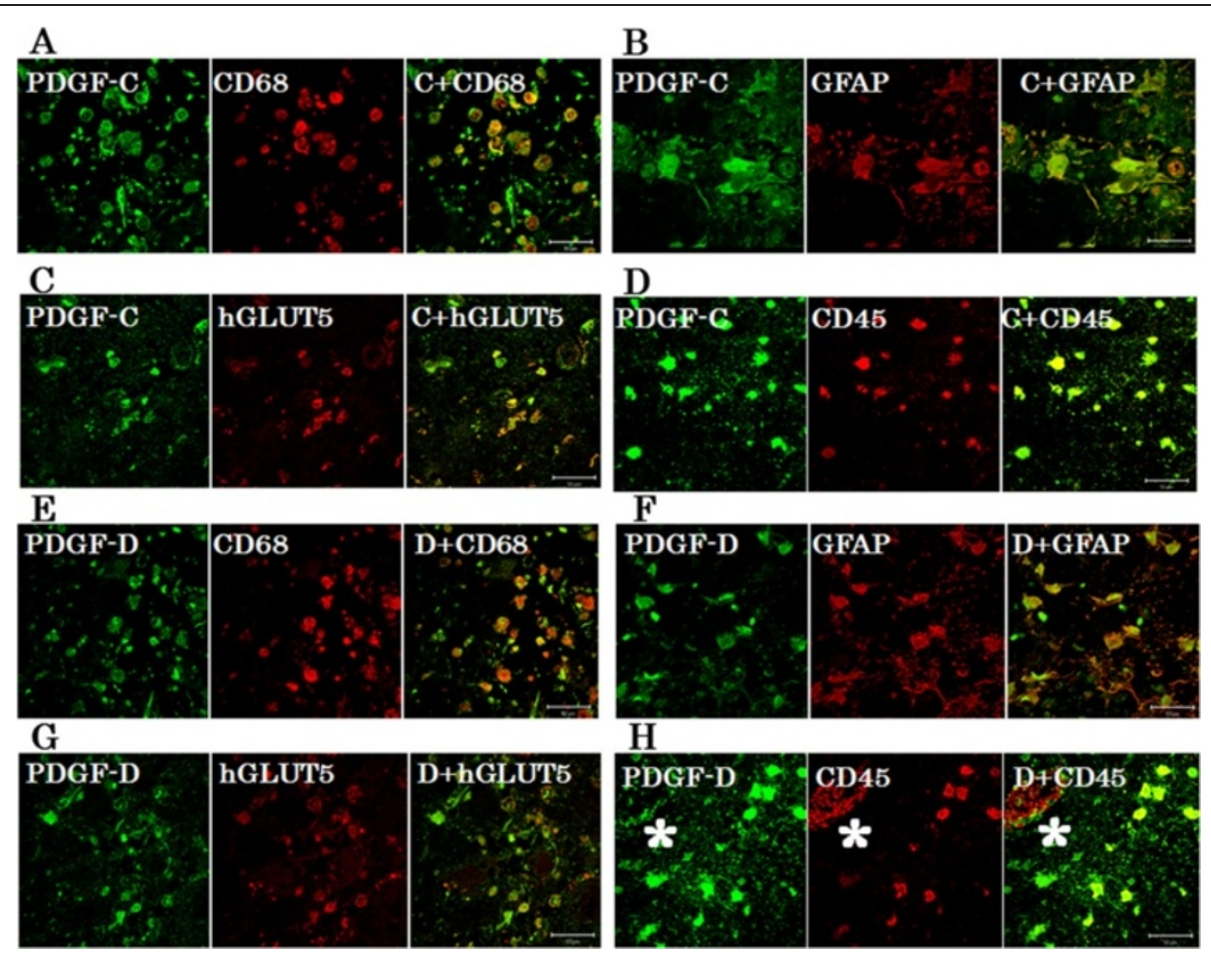

Figure 4 Double immunofluorescence staining. The results of double immunofluorescence staining from case 1 revealed that PDGF-C or D-positive cells were merged with many CD68 (A, E), GFAP (B, F), hGLUT5 (C, G), and CD45 (D, H) -positive cells in PN. Some PDGF-C or D-positive cells did not express CD68, GFAP, hGLUT5 or CD45 and vice versa. Endothelial cells (*) were nonspecifically stained with secondary fluorescence antibody. The scale bar represents $50 \mu \mathrm{m}$.

PDGFs has adverse effects. Previous studies also have demonstrated that various cell types, including macrophages, fibroblasts, pericytes, and capillary endothelial cells, express PDGFs [20,21]. Deuel et al. also reported that a macrophage-derived PDGF induces chemotaxis and the proliferation of monocytes and fibroblasts during inflammation and wound repair [22].

This is the first study to explore the expression of PDGF isoforms and PDGFRs in human brain RN. Our results have shown that all PDGFs and PDGFRs were expressed in brain RN, and that PDGFs and PDGFR $\alpha$ were primarily expressed by macrophages, microglia, reactive astrocytes, lymphocytes, and endothelial cells in PN. These findings suggest that the activation of PDGFs is coincident with inflammation, angiogenesis, and fibrogenesis in the pathophysiology of RN.

Our recent study revealed that CD45-positive lymphocytes expressing CXCR4 might be drawn into PN from peripheral blood by chemotaxis, but they do not express proinflammatory cytokines, and their roles in $\mathrm{RN}$ remain unclear (submitted for publication). However, in the present study, CD45-positive lymphocytes produced PDGF-C and -D. These results suggest that CD45-positive lymphocytes in PN do not produce proinflammatory cytokines but may play significant indirect roles in angiogenesis and/or inflammation.
The highest differences of expression among PDGFs on brain RN were observed in PDGF-C and D (Figure 3 and Additional file 4). In this study, the expressions of PDGF-C and D were significantly higher than the expressions of PDGF-A and B in PN. Our current immunohistochemical study has further revealed that inflammatory cells, including macrophages, microglia, and even lymphocytes, were gathered in PN and produced PDGF-C and D. These mononuclear cells are known to play important roles in wound healing and inflammatory disease by producing a variety of growth factors and cytokines [23,24]. In our recent study, these mononuclear cells produced inflammatory cytokines (IL-1 $\alpha$, IL-6, TNF- $\alpha$, NFkB) (submitted for publication). In the present study, these cells also produced PDGF-C and D. Therefore the activation of PDGF-C and D is coincident with inflammation as well as angiogenesis. These findings suggest that PDGF-C and D are involved in multiple aspects of brain RN.

The present and previous reports have revealed that the differential expression of PDGFs has also been seen in pathological conditions other than RN. In the aortic ring outgrowth assay, PDGF-C mediated significantly increased outgrowth, comparable to the levels mediated by VEGF and PDGF-A and B [25]. The angiogenic activity of PDGF-C in vivo is more potent than that of PDGF-A, $\mathrm{AB}$ or $\mathrm{B}[26]$. PDGF-D also has been shown to stimulate 

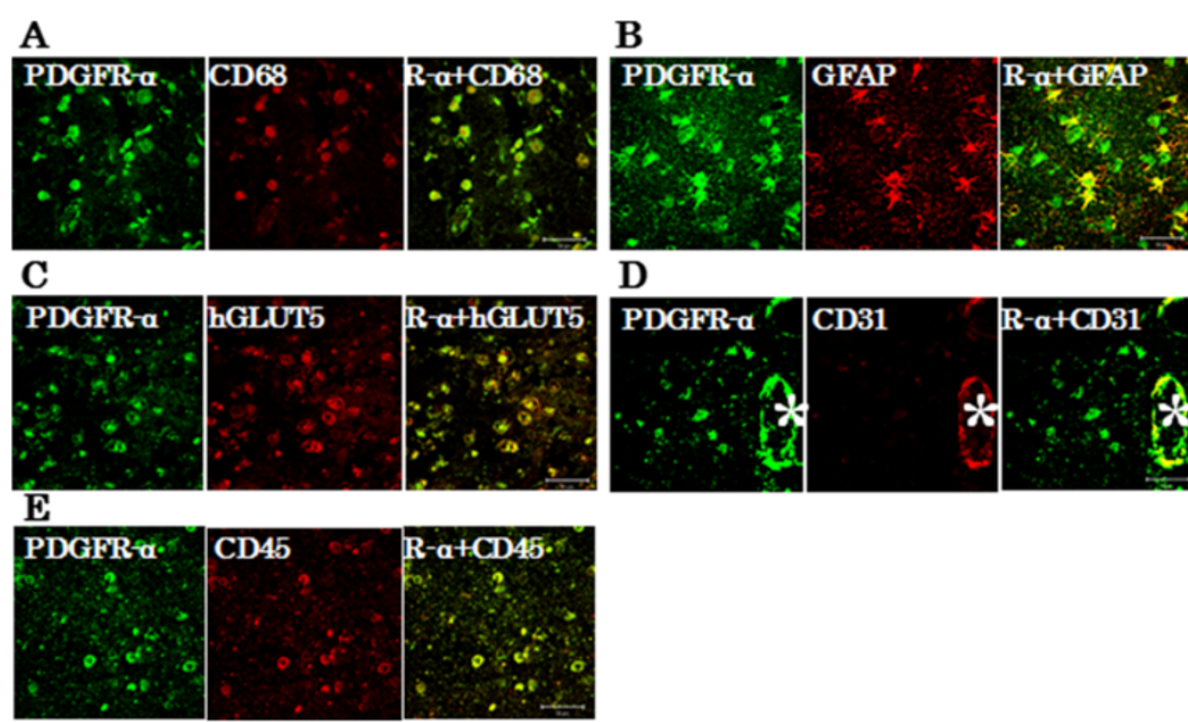

D
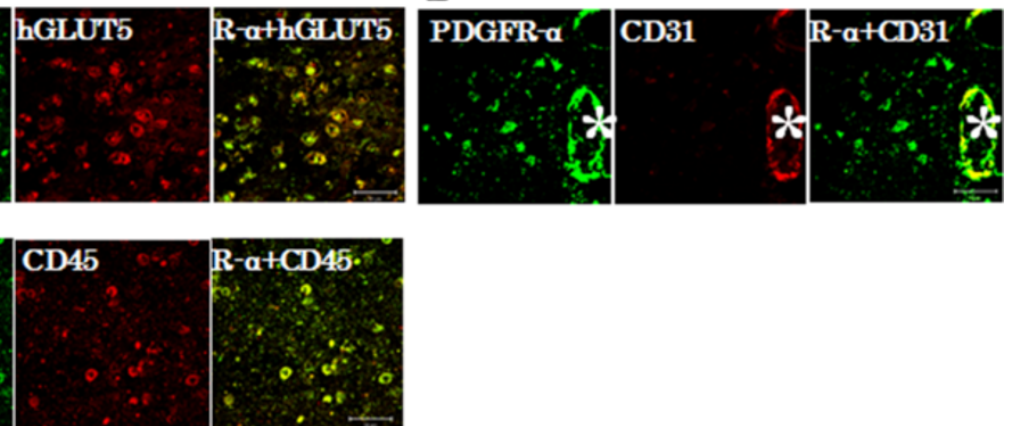

F

G
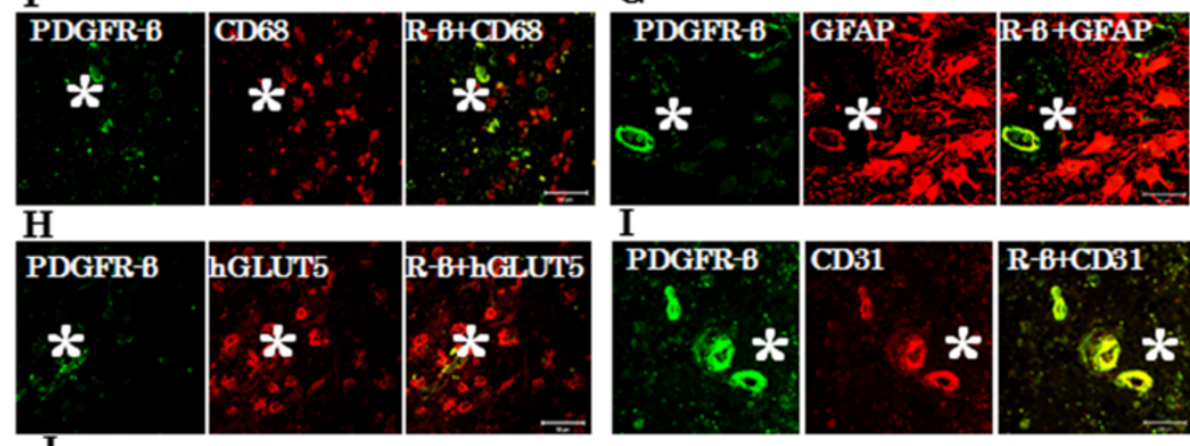

I
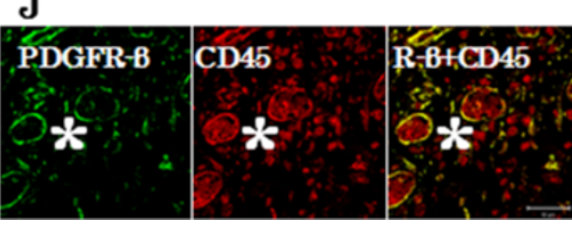

Figure 5 Double immunofluorescence staining. Double immunofluorescence staining from case 1 revealed that PDGFR-a and $\beta$ were strongly expressed in CD31-positive cells in PN (D and I). PDGFR-a positive cells were merged with many cells positive for CD68 (A), GFAP (B), hGLUT5 (C), and CD45 (E). PDGFR- $\beta$-positive cells merged specifically with endothelial cells $\left(\mathbf{F}, \mathbf{G}, \mathbf{H}, \mathbf{I}\right.$ and $\left.\mathbf{J},{ }^{*}\right)$. Endothelial cells $\left(^{*}\right)$ were nonspecifically stained with secondary fluorescence antibody. The scale bar represents $50 \mu \mathrm{m}$.

angiogenesis and to play a critical role in wound healing $[21,27,28]$.

$\mathrm{Li}$ et al. found that PDGF-D is a potent transforming and angiogenic growth factor for NIH/3 T3 cells, and that the transformed cells also induce VEGF expression [28]. Zhao et al. also found that inhibition of PDGF-D leads to decreased cell invasion in gastric cancer, partly through the regulation of VEGF [29]. In our study, many reactive astrocytes produced PDGF-C and D and expressed PDGFR- $\alpha$, but these cells did not express PDGFR- $\beta$. These results established that PDGF-C and D play roles in angiogenesis and inflammation through autocrine and paracrine stimulation. Although the functions of these isoforms of PDGFs on cells are similar in many respects, each isoform might play different roles in different cell types via various receptors and pathways.

Previously, it was reported that several types of cells participate in angiogenesis and inflammation in brain RN $[1,5,6]$. But the underlying mechanisms have not been completely elucidated. We desperately need to know why different types of cells, including macrophages, microglia, lymphocytes, and astrocytes, acquire the capacity for differentiation, producing inflammatory cytokines and growth factors under certain pathological conditions. Ungvari et al. reported that $\gamma$-irradiated cerebromicrovascular endothelial cells acquired a senescence-associated secretory phenotype (SASP) characterized by the upregulation of proinflammatory cytokines and chemokines [30]. 
Our results suggest that several types of cells that survived irradiation in PN acquired SASP, and that this mechanism may be a key process in brain RN.

In this study, we performed retrospective analysis with clinical specimens of symptomatic $\mathrm{RN}$ and revealed that PDGFs/PSGFRs were involved in RN. However, this analysis covers just one aspect of RN. It is impossible to determine whether PDGFs exacerbate $\mathrm{RN}$ or rather are produced as a byproduct of RN. Also, we cannot speculate as to the dose-response relationship or the time course of the expression of PDGFs and their receptors in RN. These questions will be answered if a reproducible animal model of $\mathrm{RN}$ can be established.

\section{Conclusions}

In conclusion, PDGFs/PDGFRs play critical roles in angiogenesis and possibly in inflammation, and they contribute to the pathogenesis of $\mathrm{RN}$, irrespective of the original tumor pathology and applied radiation modality. Moreover, the autocrine or paracrine signaling of PDGFs also plays crucial roles in aggressive angiogenesis and inflammation in RN. PDGF-C, PDGF-D and PDGFR- $\alpha$ have clinical importance, because PDGFR- $\beta$ was expressed even in UB. Treatments to inhibit PDGF-C and D, or to inhibit PDGF-C and D in combination with PDGFR- $\alpha$ with a kinase inhibitor, may provide new approaches for $\mathrm{RN}$ induced by common radiation therapies, including XRT, SRS and BNCT.

\section{Additional files}

Additional file 1: Typical MRI of symptomatic radiation necrosis from case 3. Gd-enhanced T1 MRI just prior to excision of necrotic foci (A). Gd-enhanced T1 MRI 2 weeks after surgery (A'). FLAIR MRI just prior to excision of necrotic foci (B). FLAIR MRI, 2 weeks after surgery (B'). After surgical resection of the only enhanced lesion, perilesional edema was decreased compared with preoperative MRI.

Additional file 2: Representative immunohistochemistry from case 1. Immunostaining revealed the necrotic core (A, D NC) and perinecrotic area (A, D PN). PDGF-A (A, B, C) and PDGF-B (D, E) were produced by some monocytic cells ( $B, E$ arrow) and endothelial cells $\left(C, E^{*}\right)$ in PN. Original magnification, A, D × 40, B, C, E× 200.

Additional file 3: H\&E staining and immunohistochemistry from case 3. H\&E staining (A) and immunohistochemistry (B through $O$ ) from case 3, showing NC and PN. PDGF-A (B, C) and PDGF-B (D, E) were produced by some monocytic cells (arrows in $\mathrm{C}, \mathrm{E}$ ) in PN. In contrast, PDGF-C ( $F, G, H)$ and PDGF-D (I, J) were produced by many monocytic cells (arrows in $G, H, J$ ), reactive astrocytic cells (arrowheads in $G$, J), and endothelial cells $\left(H, J^{*}\right)$. PDGFR-a $(K, L, M)$ was expressed in monocytic cells ( $L$, arrow), reactive astrocytic cells ( $L$, arrowhead) and endothelial cells $\left(M^{*}\right)$ in PN. PDGFR- $\beta(N, O)$ was expressed mainly in endothelial cells (O*). Original magnification, A, B, D, F, I, K, N×40, C, E, G, H, J, L, M, O $\times 200$.

Additional file 4: Frequency of expression of PDGFs in the GBM group and non-GBM group. We assessed the frequency of expression of PDGFs semi-quantitatively. In the GBM group (cases 3, 4, 5, 6) and non-GBM group (cases $1,2,7$ ), there was no apparent statistical significance in expression of each isoform (A, B, C, D).
Additional file 5: Double immunofluorescence staining results from case 3. Double immunofluorescence staining from case 3 revealed that PDGF-C or D-positive cells were merged with many CD68, hGLUT5, CD45 and GFAP-positive cells. Endothelial cells $\left(^{*}\right)$ were nonspecifically stained with secondary fluorescence antibody. The scale bar represents $50 \mu \mathrm{m}$.

Additional file 6: Double immunofluorescence staining results from case 3. Double immunofluorescence staining of the specimen from case 3 revealed that PDGFR- $\alpha$ and $\beta$ were strongly expressed in CD31-positive cells ( $D$ and I). PDGFR-a-positive cells were merged with many cells positive for CD68 (A), GFAP (B), hGLUT5 (C), and CD45 (E). PDGFR- $\beta$-positive cells were merged specifically with endothelial cells ( $F$ thorough J). Endothelial cells $\left(^{*}\right)$ were nonspecifically stained with secondary fluorescence antibody. The scale bar represents $50 \mu \mathrm{m}$.

Additional file 7: Immunofluorescence staining from consecutive specimens from case 1 and 3 . Immunofluorescence staining of consecutive specimens from case $1(A, B)$ and $3(C, D)$ showed positivity for PDGFR- $\beta$ (A) or GFAP (B). PDGFR- $\beta$ (A) was not observable at an excitation wavelength of $561 \mathrm{~nm}$ but was apparent at $499 \mathrm{~nm}$ in endothelial cells (*). On the other hand, GFAP (B) was observed only at an excitation wavelength of $561 \mathrm{~nm}$ in reactive astrocytes. The scale bar represents $50 \mu \mathrm{m}$.

\section{Abbreviations}

RN: Radiation necrosis; PDGFs: Platelet-derived growth factors; PDGFRs: Platelet-derived growth factor receptors; H\&E: Hematoxylin and eosin; PN: Perinecrotic area; UB: Undamaged brain tissue; VEGF: Vascular endothelial growth factor; HIF-1a: Hypoxia-inducible factor 1a; XRT: X-ray treatment; SRS: Stereotactic radiosurgery; Proton: Proton beam therapy; BNCT: Boron neutron capture therapy; NC: Necrotic core; SASP: Senescenceassociated secretory phenotype; CTLs: Cytotoxic T-lymphocytes.

\section{Competing interests}

The authors declare that they have no competing interests.

\section{Authors' contributions}

TM carried out the statistical analysis and drafted the manuscript. S-IM conceived of the study, participated in its design and coordination, and helped to draft the manuscript. TT, NN, MF, HK, EY, SK, and TK participated in the study design and coordination and helped to draft the manuscript. All authors read and approved the final manuscript.

\section{Acknowledgments}

This work was partly supported by a Grant-in-Aid for Scientific Research (B) (23390355) and by a Grant-in-Aid for Exploratory Research (24659658) to S-I. M. and by a Grant-in-Aid for Scientific Research (C) (23592145) to M.F. from the Japanese Ministry of Education, Culture, Sports, Science, and Technology. We thank Itsuko Inoue and Kaname Shimokawa for their technical assistance.

\section{Author details}

'Department of Neurosurgery, Osaka Medical College, 2-7 Daigaku-machi, Takatsuki City, Osaka 569-8686, Japan. ${ }^{2}$ Department of Pathology, Osaka Medical College, 2-7 Daigaku-machi, Takatsuki City, Osaka 569-8686, Japan.

Received: 15 October 2013 Accepted: 3 February 2014

Published: 11 February 2014

\section{References}

1. Nonoguchi N, Miyatake S, Fukumoto M, Furuse M, Hiramatsu R, Kawabata S, Kuroiwa T, Tsuji M, Ono K: The distribution of vascular endothelial growth factor-producing cells in clinical radiation necrosis of the brain: pathological consideration of their potential roles. J Neurooncol 2011, 105:423-431.

2. Giglio P, Gilbert MR: Cerebral radiation necrosis. Neurologist 2003, 9:180-188.

3. Glantz MJ, Burger PC, Friedman AH, Radtke RA, Massey EW, Schold SC Jr: Treatment of radiation-induced nervous system injury with heparin and warfarin. Neurology 1994, 44:2020-2027.

4. Levin VA, Bidaut L, Hou P, Kumar AJ, Wefel JS, Bekele BN, Grewal J, Prabhu S, Loghin M, Gilbert MR, Jackson EF: Randomized double-blind placebo-controlled trial of bevacizumab therapy for radiation necrosis of the central nervous system. Int J Radiat Oncol Biol Phys 2011, 79:1487-1495. 
5. Furuse M, Nonoguchi N, Kawabata S, Yoritsune E, Takahashi M, Inomata T, Kuroiwa T, Miyatake S: Bevacizumab treatment for symptomatic radiation necrosis diagnosed by amino acid PET. Jpn J Clin Oncol 2013, 43:337-341.

6. Miyatake SI, Furuse M, Kawabata S, Maruyama T, Kumabe T, Kuroiwa T, Ono K: Bevacizumab treatment of symptomatic pseudoprogression after boron neutron capture therapy for recurrent malignant gliomas. Report of 2 cases. Neuro-oncology 2013, 15:650-655.

7. Furuse M, Kawabata S, Kuroiwa T, Miyatake S: Repeated treatments with bevacizumab for recurrent radiation necrosis in patients with malignant brain tumors: a report of 2 cases. J Neurooncol 2011, 102:471-475.

8. Arbab AS, Janic B, Jafari-Khouzani K, Iskander AS, Kumar S, Varma NR, Knight RA, Soltanian-Zadeh H, Brown SL, Frank JA: Differentiation of glioma and radiation injury in rats using in vitro produce magnetically labeled cytotoxic T-cells and MRI. PLoS One 2010, 5:e9365.

9. Kumar S, Arbab AS, Jain R, Kim J, deCarvalho AC, Shankar A, Mikkelsen T, Brown SL: Development of a novel animal model to differentiate radiation necrosis from tumor recurrence. J Neurooncol 2012, 108:411-420.

10. Heldin $\mathrm{CH}$, Westermark B: Mechanism of action and in vivo role of platelet-derived growth factor. Physiol Rev 1999, 79:1283-1316.

11. Heldin $\mathrm{CH}$, Ostman A, Ronnstrand L: Signal transduction via plateletderived growth factor receptors. Biochim Biophys Acta 1998, 1378:F79-F113.

12. Bergsten E, Uutela M, Li X, Pietras K, Ostman A, Heldin CH, Alitalo K, Eriksson U: PDGF-D is a specific, protease-activated ligand for the PDGF beta-receptor. Nat Cell Biol 2001, 3:512-516.

13. Li X, Ponten A, Aase K, Karlsson L, Abramsson A, Uutela M, Backstrom G, Hellstrom M, Bostrom H, Li H, et al: PDGF-C is a new protease-activated ligand for the PDGF alpha-receptor. Nat Cell Biol 2000, 2:302-309.

14. Wang Z, Ahmad A, Li Y, Kong D, Azmi AS, Banerjee S, Sarkar FH: Emerging roles of PDGF-D signaling pathway in tumor development and progression. Biochim Biophys Acta 1806, 2010:122-130.

15. Lei $H$, Kazlauskas $A$ : Focus on molecules: platelet-derived growth factor $C$, PDGF-C. Exp Eye Res 2008, 86:711-712.

16. Wang Z, Kong D, Banerjee S, Li Y, Adsay NV, Abbruzzese J, Sarkar FH: Downregulation of platelet-derived growth factor- $D$ inhibits cell growth and angiogenesis through inactivation of Notch-1 and nuclear factor-kappaB signaling. Cancer Res 2007, 67:11377-11385.

17. Hoch RV, Soriano P: Roles of PDGF in animal development. Development 2003, 130:4769-4784.

18. Li R, Maminishkis A, Wang FE, Miller SS: PDGF-C and -D induced proliferation/ migration of human RPE is abolished by inflammatory cytokines. Invest Ophthalmol Vis Sci 2007, 48:5722-5732.

19. Lindahl P, Johansson BR, Leveen P, Betsholtz C: Pericyte loss and microaneurysm formation in PDGF-B-deficient mice. Science 1997, 277:242-245.

20. Li X, Kumar A, Zhang F, Lee C, Li Y, Tang Z, Arjuna P: VEGF-independent angiogenic pathways induced by PDGF-C. Oncotarget 2010, 1:309-314.

21. Uutela M, Wirzenius M, Paavonen K, Rajantie I, He Y, Karpanen T, Lohela M, Wiig $H$, Salven P, Pajusola K, et al: PDGF-D induces macrophage recruitment, increased interstitial pressure, and blood vessel maturation during angiogenesis. Blood 2004, 104:3198-3204.

22. Deuel TF, Senior RM, Huang JS, Griffin GL: Chemotaxis of monocytes and neutrophils to platelet-derived growth factor. J Clin Invest 1982, 69:1046-1049.

23. Kumagai $S$, Ohtani $H$, Nagai T, Funa K, Hiwatashi N, Shimosegawa T, Nagura h: Platelet-derived growth factor and its receptors are expressed in areas of both active inflammation and active fibrosis in inflammatory bowel disease. Tohoku J Exp Med 2001, 195:21-33.

24. Leibovich SJ, Ross R: The role of the macrophage in wound repair. A study with hydrocortisone and antimacrophage serum. Am J Pathol 1975, 78:71-100.

25. Gilbertson DG, Duff ME, West JW, Kelly JD, Sheppard PO, Hofstrand PD, Gao Z, Shoemaker K, Bukowski TR, Moore M, et al: Platelet-derived growth factor C (PDGF-C), a novel growth factor that binds to PDGF alpha and beta receptor. J Biol Chem 2001, 276:27406-27414.

26. Cao R, Brakenhielm E, Li X, Pietras K, Widenfalk J, Ostman A, Eriksson U, Cao $Y$ : Angiogenesis stimulated by PDGF-CC, a novel member in the PDGF family, involves activation of PDGFR-alphaalpha and -alphabeta receptors. FASEB J 2002, 16:1575-1583.

27. LaRochelle WJ, Jeffers M, Corvalan JR, Jia XC, Feng X, Vanegas S, Vickroy JD, Yang XD, Chen F, Gazit G, et al: Platelet-derived growth factor D: tumorigenicity in mice and dysregulated expression in human cancer. Cancer Res 2002, 62:2468-2473.

28. Li H, Fredriksson L, Li X, Eriksson U: PDGF-D is a potent transforming and angiogenic growth factor. Oncogene 2003, 22:1501-1510.

29. Zhao L, Zhang C, Liao G, Long J: RNAi-mediated inhibition of PDGF-D leads to decreased cell growth, invasion and angiogenesis in the SGC-7901 gastric cancer xenograft model. Cancer Biol Ther 2010, 9:42-48.

30. Ungvari Z, Podlutsky A, Sosnowska D, Tucsek Z, Toth P, Deak F, Gautam T, Csiszar A, Sonntag WE: lonizing radiation promotes the acquisition of a senescence-associated secretory phenotype and impairs angiogenic capacity in cerebromicrovascular endothelial cells: role of increased DNA damage and decreased DNA repair capacity in microvascular radiosensitivity. J Geronto/ Series A, Biol Sci Med Sci 2013, 68:1443-1457.

doi:10.1186/1748-717X-9-51

Cite this article as: Miyata et al:: The roles of platelet-derived growth factors and their receptors in brain radiation necrosis. Radiation Oncology 2014 9:51.

\section{Submit your next manuscript to BioMed Central and take full advantage of:}

- Convenient online submission

- Thorough peer review

- No space constraints or color figure charges

- Immediate publication on acceptance

- Inclusion in PubMed, CAS, Scopus and Google Scholar

- Research which is freely available for redistribution 